\title{
Frequency-shift free optical phase conjugation using counter-propagating dual pump four-wave mixing in fiber
}

\author{
Abhishek Anchal ${ }^{1,4}$, Pradeep Kumar $\mathrm{K}^{1,2}$ and Pascal Landais ${ }^{3}$ \\ ${ }^{1}$ Department of Electrical Engineering, Indian Institute of Technology Kanpur, Kanpur, 208016, India \\ ${ }^{2}$ Center for Laser and Photonics, Indian Institute of Technology Kanpur, Kanpur, 208016, India \\ ${ }^{3}$ School of Electronic Engineering, Dublin City University, Glasnevin, Dublin 9, Ireland
}

\section{Introduction}

Optical phase conjugation (OPC) finds variety of applications in waveform reconstruction, nonlinear spectroscopy, phase locking, interferometry, optical data processing, optical squeezing, dispersion and nonlinearity compensation in single mode fibers [1-4]. OPC is generated through various processes e.g. three and four-wave mixing in periodically poled $\mathrm{LiNbO}_{3}$ crystal, highly nonlinear fibers (HNLFs) or semiconductor optical amplifiers (SOAs) [1, 5-7]. In particular, HNLF is easily spliced to single mode fiber with low loss and hence, OPC in HNLF is specifically suitable for telecom applications like dispersion and nonlinearity compensation via mid span spectral inversion (MSSI) [8]. In MSSI, impairments due to fiber dispersion and nonlinearities in the first half of the fiber are inverted by OPC, and compensated by subsequent transmission through the second half of the fiber. Nonlinear compensation based on MSSI using OPC in HNLF and SOA is also been demonstrated in [4, 9]. However, in this method, OPC is accompanied by shift in signal frequency leading to strong channel crosstalk in WDM systems. Another method based on OPC in silicon waveguides has been demonstrated [10]. This method shows low channel crosstalk and hence can be used in WDM systems, but requires additional optical amplifiers to compensate for the 
optical losses in silicon waveguide. It also requires careful selection of waveguide dimensions to achieve phase matching and higher conversion efficiency.

Several approaches have been reported for generation of non-frequency shifted OPC, which includes co-propagating orthogonally polarized dual pump FWM in SOA and fibers $[11,12]$. However, all the techniques cited above require the polarization state of signal to be parallel with one of the pumps, results in shift of polarization of conjugated signal. Since, polarization of signal in a transmission system cannot be readily controlled, so a technique for polarization insensitive frequency-shift free $\mathrm{OPC}$ is required.

In this paper, we propose a new technique to realize polarization insensitive frequency-shift free phase conjugation. OPC is achieved by counter-propagating dual pump FWM in HNLF, in which first pump and signal waves are injected into one end of the HNLF and a second pump is injected into other end. The two counter-propagating pumps creates standing wave which results in Bragg grating throughout the length of HNLF. The incoming signal diffracts from this grating and generates idler. The generated idler propagates in the direction opposite to that of the signal. We place the pump frequencies symmetrical about signal frequency such that the generated idler has same frequency as that of the signal. This symmetric placement of pump frequencies allows for large separation between pump and signal frequencies and yet a small phase mismatch. The small phase mismatch causes the efficient generation of idler. We show analytically that idler phase is conjugate of signal phase for optimum fiber length. The signal and idler are spatially separated and extracted at opposite ends of the fiber. We demonstrate that our proposed OPC model is able to generate perfect phase conjugation, by modulating the signal with a non-zero chirp intensity modulator and resolving the temporal profile of electrical field envelope of idler. We further investigate the effects of length of HNLF and pump power on efficiency and phase-offset of OPC.

A scheme describing backward generated idler at different frequency, but whose phase is conjugate of signal was proposed in [13], in which, two forward propagating pump interact with co-propagating signal to generate an idler which is non-conjugates and frequency shifted image of signal. A third forward propagating pump, together with one of the initial pumps, signal and idler interact via FWM process to generate phase conjugation. In $[11,12]$, frequency-shift free phase conjugated wave was co-propagated with signal and shift of polarization in conjugated wave assisted the filtering of conjugate. However in our scheme, frequency-shift free phase conjugated wave and signal wave appears at opposite end of fiber and hence spatially filtered, thus eliminating the need of polarization-shift in conjugate.

The advantages of our proposed technique are: (a) generation of phase conjugated wave (idler) at C-band, (b) no frequency and polarization shift in conjugated wave (idler), and (c) polarization insensitive phase conjugation. We analyze signal and idler evolution in the fiber using nonlinear Schrodinger equation (NLSE) formalism under strong pump approximation. The resulting coupled differential equations

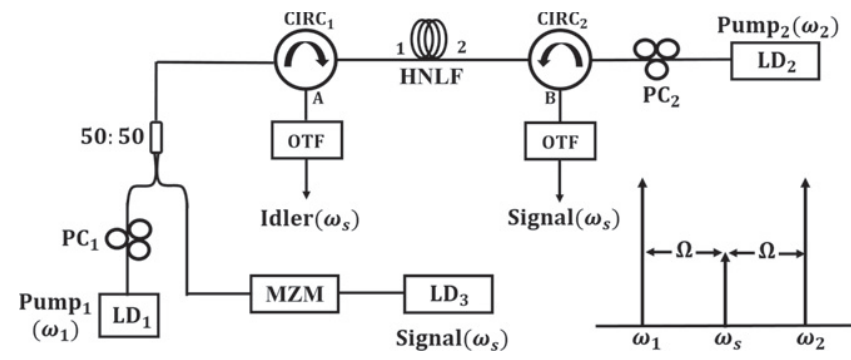

Figure 1. Proposed scheme for optical phase conjugation in nonlinear fiber. The two pumps propagate in opposite directions as do signal and idler waves. Phase of idler wave is conjugate of that of signal wave. The phase conjugated signal (idler) wave appears at fiber input $z=0$ while the residual signal appears at fiber output $z=L$. Symmetrical placement of pump frequencies allow efficient generation of idler at signal frequency. $\mathrm{HNLF}=$ highly nonlinear fiber, $\mathrm{PC}_{1,2}=$ polarization controller, $\mathrm{CIRC}_{1,2}=$ circulator, $\mathrm{LD}_{1,2,3}=$ laser diode, $\mathrm{MZM}=$ Mach-Zehnder modulator, $\mathrm{OTF}=$ optical tunable filter.

are numerically solved using Runge-Kutta 45 (rk45) method and the results are compared with analytical solution to validates our simulation. We observe perfect phase conjugation with efficiency $-17 \mathrm{~dB}$ for HNLF length of $2.85 \mathrm{~km}$ and pumps power at $9.22 \mathrm{dBm}$.

The rest of the paper is organized as follows; in section 2 we briefly discuss the theory of counter-propagating dual pump FWM in HNLF, leading to OPC generation. In section 3 , we discuss simulation results of our proposed scheme of phase conjugation. Finally, we conclude by summarizing our results in section 4 .

\section{System description}

Figure 1 shows the schematic diagram of the proposed method to generate OPC. The frequencies $\omega_{1}, \omega_{2}$, and $\omega_{\mathrm{s}}$ corresponds to pump $_{1}$, pump p $_{2}$, and signal respectively. Pump and signal waves are fed into one end on HNLF and pump $p_{2}$ is fed into other end. The two counter-propagating pump forms standing wave inside HNLF and which in turn creates Bragg grating. This grating diffracts signal to create idler at same frequency i.e. at $\omega_{\mathrm{s}}$. This process must satisfy frequency and phase matching conditions: $\omega_{1}+\omega_{2}=2 \omega_{\mathrm{s}}$ and $\mathbf{k}_{1}+\mathbf{k}_{2}=\mathbf{k}_{\mathrm{s}}+\mathbf{k}_{\mathrm{i}}$, where $\mathbf{k}_{1,2}, \mathbf{k}_{\mathrm{s}}$, and $\mathbf{k}_{\mathrm{i}}$ denote propagation vectors of pumps, signal, and idler respectively. Since signal and idler waves are counterpropagating $\left(\mathbf{k}_{\mathrm{s}}=-\mathbf{k}_{\mathrm{i}}\right)$ phase matching condition reduces to $\mathbf{k}_{1}+\mathbf{k}_{2}=0$. Although the two pump waves are counterpropagating and hence, have opposite signs of $\mathbf{k}$, their magnitudes are not equal. Hence a residual phase mismatch $\Delta k=\left|\mathbf{k}_{1}\right|-\left|\mathbf{k}_{2}\right| \neq 0$ must be taken into account while deriving expressions governing signal and idler evolution in the fiber. We choose $\omega_{1}=\omega_{\mathrm{s}}-\Omega$ and $\omega_{2}=\omega_{\mathrm{s}}+\Omega$ to minimize residual phase mismatch $\Delta k$. To further minimize $\Delta k$, we take the frequencies of signal and idler to be zero dispersion frequency of the fiber. Expanding $\Delta k$ at $\omega_{\mathrm{s}}$ using Taylor's series, we obtain, $\Delta k=2 k^{(1)} \Omega$, where $k^{(1)}=\left.\frac{\partial k}{\partial \omega}\right|_{\omega=\omega_{\mathrm{s}}}$, shows that the residual phase mismatch 
$(\Delta k)$ depends upon the detuning between pumps and signal $(\Omega)$.

The electric field of the two pumps, signal, and idler waves are given by $A_{1} \exp \left(j\left(\omega_{1} t-k_{1} z\right)\right)$, $A_{2} \exp \left(j\left(\omega_{2} t-k_{2} z\right)\right), \quad A_{\mathrm{s}} \exp \left(j\left(\omega_{\mathrm{s}} t-k_{\mathrm{s}} z\right)\right), \quad$ and $A_{\mathrm{i}} \exp \left(j\left(\omega_{\mathrm{i}} t-k_{\mathrm{i}} z\right)\right)$ respectively, where $A_{1}, A_{2}, A_{\mathrm{s}}$, and $A_{\mathrm{i}}$ are slow varying pulse envelopes. Assuming strong pump approximation and that all waves are co-polarized (for simplicity), equations governing signal and idler evolution can be obtained by NLSE [14]

$$
\frac{\partial E}{\partial z}=-k^{(1)} \frac{\partial E}{\partial t}+j \frac{k^{(2)}}{2} \frac{\partial^{2} E}{\partial t^{2}}-j \gamma|E|^{2} E,
$$

where $E$ is the total electric field and $\gamma$ is the nonlinear coefficient of the fiber. Substituting $E=A_{1} \exp \left(j\left(\omega_{1} t-k_{1} z\right)\right)+A_{2} \exp \left(j\left(\omega_{2} t-k_{2} z\right)\right)$

$+A_{\mathrm{s}} \exp \left(j\left(\omega_{\mathrm{s}} t-k_{\mathrm{s}} z\right)\right)+A_{\mathrm{i}} \exp \left(j\left(\omega_{\mathrm{i}} t-k_{\mathrm{i}} z\right)\right)$ into (1), and simplifying we obtain

$$
\begin{aligned}
\frac{\partial A_{\mathrm{s}, \mathrm{i}}(z, t)}{\partial z}= & -\frac{\alpha}{2} A_{\mathrm{s}, \mathrm{i}}+j \gamma\left(\left|A_{\mathrm{s}, \mathrm{i}}^{2}\right|+2\left|A_{\mathrm{i}, \mathrm{s}}^{2}\right|\right) A_{\mathrm{s}, \mathrm{i}} \\
& +2 j \gamma \sum_{p=1,2}\left|A_{p}^{2}\right| A_{\mathrm{s}, \mathrm{i}} \pm 2 j \gamma A_{1} A_{2} A_{\mathrm{i}, \mathrm{s}}^{*} e^{-j \Delta k z},
\end{aligned}
$$

where $\alpha$ is fiber attenuation. In (2), the first, second, and third term accounts for attenuation, self phase modulation (SPM), and cross phase modulation (XPM) respectively, and fourth term accounts for FWM. The evolution of signal and idler in equation (2) is derived in a similar way of signal and idler evolution for forward FWM in HNLF derived in [14]. We keep the detuning between pumps and signal $(\Omega)$ away from gain range of stimulated Brillouin and Raman scattering processes to neglect the contribution of Brillouin and Raman scattering.

Analytical solution of (2) is complex and to simplify the solution we neglect the attenuation, SPM, and XPM in a first instance. Solving (2) subject to boundary conditions, $A_{\mathrm{s}}(0)=A_{s 0}$ and $A_{\mathrm{i}}(L)=0$, we obtain

$$
\begin{aligned}
A_{\mathrm{s}}(z)= & \frac{1}{\zeta} \exp (-j \Delta k z / 2)[\{\mu \cos (\mu(z-L)) \\
& +(j \Delta k / 2) \sin (\mu(z-L))\}] A_{\mathrm{s} 0} \\
A_{\mathrm{i}}(z)= & -\frac{j}{\zeta^{*}} \kappa^{*} \sin (\mu(z-L)) \exp (-j \Delta k z / 2) A_{\mathrm{s} 0}^{*},
\end{aligned}
$$

where $\sqrt{|\kappa|^{2}+(\Delta k / 2)^{2}} \zeta=\mu \cos (\mu L)-j(\Delta k / 2) \sin (\mu L), \mu=$ $\sqrt{|\kappa|^{2}+(\Delta k / 2)^{2}}$, and $\kappa=2 \gamma A_{1}^{*} A_{2}^{*}$. The idler phase $\phi_{\mathrm{i}}(z)$ calculated from (3) is

$$
\phi_{\mathrm{i}}(z)=\frac{\Delta k z}{2}+\frac{\pi}{2}-\tan ^{-1}\left(\frac{\Delta k}{2 \mu} \tan (\mu L)\right)+\phi_{\mathrm{s}}^{*}(0),
$$

where $\phi_{\mathrm{s}}(0)$ is the phase of the signal wave at $z=0$. At fiber input, $\phi_{\mathrm{i}}(0)=\frac{\pi}{2}-\tan ^{-1}\left(\frac{\Delta k}{2 \mu} \tan (\mu L)\right)+\phi_{\mathrm{s}}^{*}(0)$; for perfect phase conjugation $\phi_{\mathrm{i}}$ must be conjugate of signal phase i.e. $\phi_{\mathrm{i}}(0)=\phi_{s}^{*}(0)$. We define phase-offset $\left(\Delta \Phi_{\text {offset }}\right)$ as the extra phase of the idler in addition to inverted signal phase:

$$
\Delta \Phi_{\text {offset }}=\frac{\pi}{2}-\tan ^{-1}\left(\frac{\Delta k}{2 \mu} \tan (\mu L)\right) .
$$

The phase-offset $\Delta \Phi_{\text {offset }}$, depends upon the pumps power, residual phase mismatch and length of HNLF. The HNLF length for which $\Delta \Phi_{\text {offset }}=0$ is defined as optimum fiber length $L_{\mathrm{op}}$. From (5), we find that $L_{\mathrm{op}}=\pi / 2 \mu$. We note that, the optimum fiber length is function of pumps power. We can ensure $\phi_{\mathrm{i}}(0)=\phi_{\mathrm{s}}{ }^{*}(0)$ by choosing fiber length $L$ equal to $L_{\mathrm{op}}$. Finally, we define efficiency of the idler wave as the ratio of idler power at $z=0$ to signal power at $z=0$ :

$$
\eta=\frac{\left|A_{\mathrm{i}}(0)\right|^{2}}{\left|A_{\mathrm{s}}(0)^{*}\right|^{2}}=\left|\frac{\kappa^{*}}{\zeta^{*}}\right|^{2} \sin ^{2}(\mu L) .
$$

Thus, efficiency $\eta$ is periodic function of fiber length $L$, with periodicity ' $\pi / \mu \quad\left(=2 L_{\mathrm{op}}\right)$ '. The efficiency $\eta$ and it is periodicity depends on pumps power and residual phase mismatch through $\mu$. Both the phase-offset and efficiency depends on the linewidth of pumps and signal. As linewidth of pump and signal waves increases, residual phase mismatch $\Delta k$ also increases resulting in deterioration of phase-offset and efficiency.

Finally, at $z=0$, idler is conjugate of the signal wave, if $L=L_{\text {op. }}$. The above formulation is valid for the pulse waves propagation in HNLF as the dispersion in HNLF is very small.

\section{Simulation results}

We have simulated (2) numerically using rk45 method and compared the results with theoretical expressions (3) for signal and idler evolution. To validate our simulations, we first simulate (2) neglecting the SPM and XPM contribution with $\alpha=0$. We verify that the simulation result is in close agreement with (3). Later we accounted for SPM and XPM effect with $\alpha=0.9 \mathrm{~dB} \mathrm{~km}^{-1}$ for signal and idler evolution. We choose the simulation parameter of industrial highly nonlinear fiber with zero-dispersion wavelength (ZDW) at $1540 \mathrm{~nm}$ [15]. The parameters are: pump wavelengths $\lambda_{1}=1539.5 \mathrm{~nm}$ and $\lambda_{2}=1540.5 \mathrm{~nm}$, signal and idler wavelengths $\lambda_{\mathrm{s}}\left(=\lambda_{\mathrm{i}}\right)=1540 \mathrm{~nm}, \gamma=9.3 \mathrm{~W}^{-1} \mathrm{~km}^{-1}$, signal power of $-7 \mathrm{dBm}$, and pump power of $9.22 \mathrm{dBm}$ each. We keep the two pumps power equal, to reduce FWM instability resulting from exchange of power between two pumps $[16,17]$. The optimum fiber length $L_{\text {op }}$ was calculated to be $3 \mathrm{~km}$ from the knowledge of $\mu$. We keep, $\Omega=63.25 \mathrm{GHz}$ to avoid the stimulated Brillouin and Raman scattering. Brillouin and Raman scattering processes have significant gain at $10.45-10.55 \mathrm{GHz}$ and $5-20 \mathrm{THz}$ detuning range respectively.

We investigate the phase inversion by OPC, using on-off keying modulated chirped signal, which can be generated by a non-zero chirp Mach-Zehnder modulator (MZM) with repetitive pattern of '1101' at 40 Gbps. Unbalanced optical power in MZM couplers introduces chirp in the signal. We model 


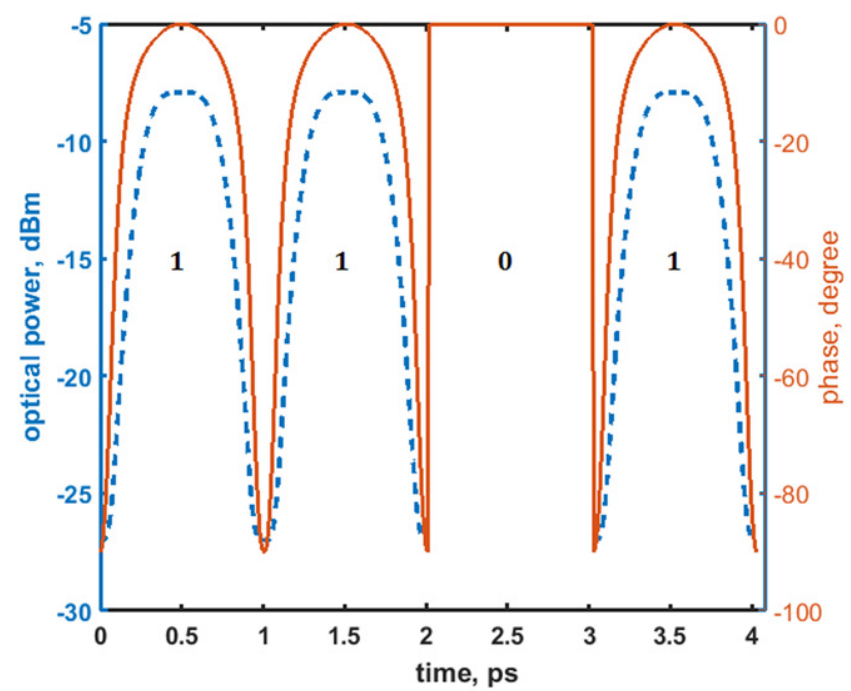

(a)

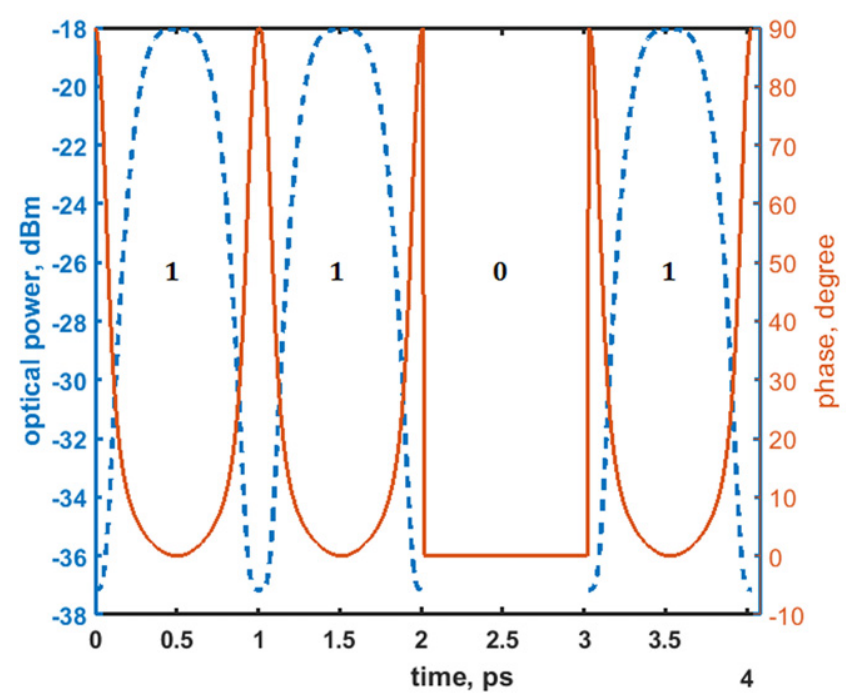

(b)

Figure 2. Intensity and phase versus time plot of (a) input signal and (b) idler. Dashed line-intensity. Solid line-phase. Phase inversion in idler wave with respect to phase of signal wave confirms the phase conjugation.

the chirping in MZM as [18]:

$$
A_{\mathrm{s}, \text { out }}=\frac{A_{\mathrm{s}}}{2}\left[e^{j \frac{\pi V_{\mathrm{RF}}}{2 V_{\pi}}}+\Gamma e^{j \frac{\pi V_{\mathrm{RF}}}{2 V_{\pi}}}\right]
$$

where $V_{\pi}=10 \mathrm{~V}$ is half-wave voltage of MZM, $V_{\mathrm{RF}}=-\frac{V_{\pi}}{2}+\frac{V_{\pi}}{2} \cos \left(\frac{2 \pi t}{T_{\mathrm{s}}}\right)$ for $50 \%$ return-to-zero pulse shaping, and $\Gamma=0.8$. Figure 2 shows plot of temporal profile of intensity and phase of signal and conjugate wave versus time. Dashed line in figure 2(a) shows intensity modulation of signal at $40 \mathrm{Gbps}$ and solid line shows the chirping introduce in signal by MZM. Figure 2(b) shows a similar intensity variation of the idler wave demonstrates modulation information transfer from signal and phase of idler wave is inverse of that of the phase of signal wave confirms phase conjugation. In this process, we neglected the pulse broadening of idler due to dispersion in HNLF, as the

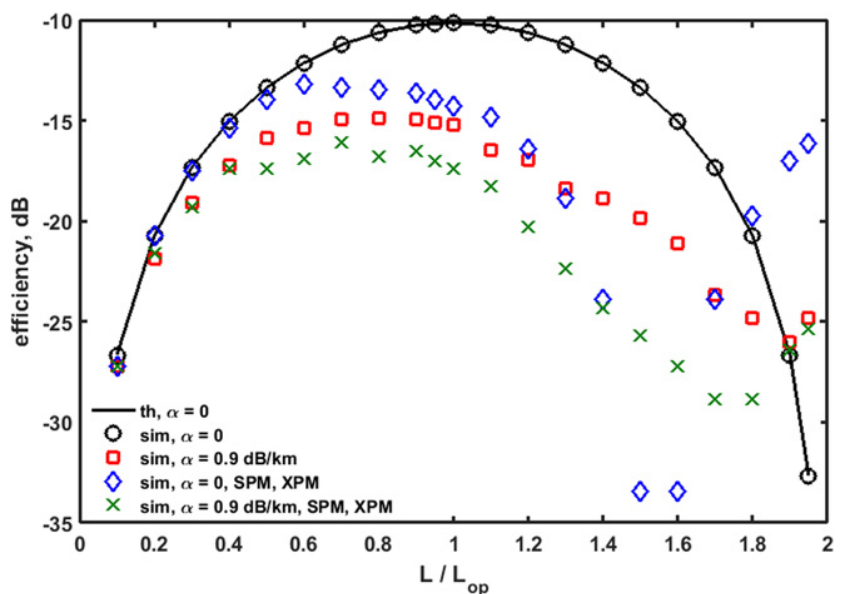

(a)

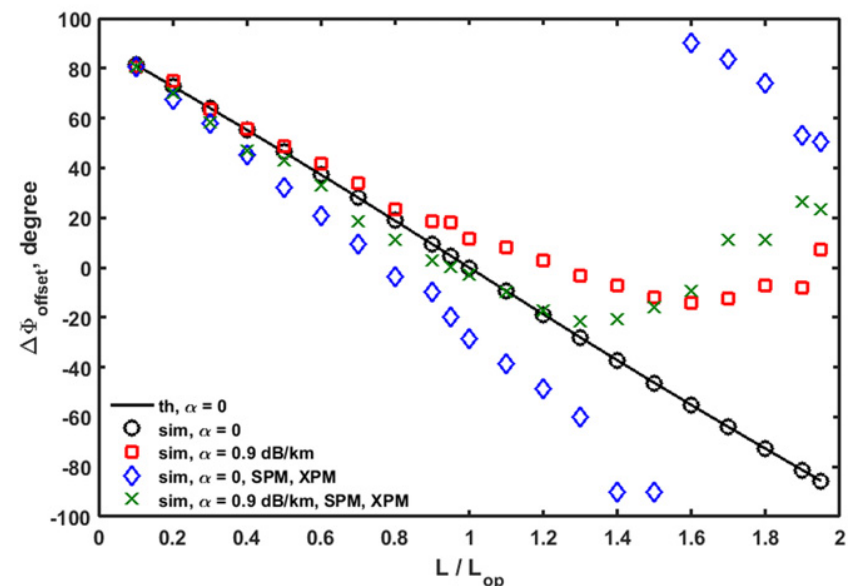

(b)

Figure 3. Effect of HNLF length on idler. In figure 3(a), efficiency increase up to optimum fiber length and then starts decreasing. In figure 3(b), variation of phase-offset with length of HNLF is shown. Signal power is kept constant at $-7 \mathrm{dBm}$ and both pump power at $9.22 \mathrm{dBm}$ with $L_{\mathrm{op}}=3 \mathrm{~km}$. For $L<L_{\mathrm{op}}$, FWM dominates over SPM and FWM and for $L>L_{\mathrm{op}}$, SPM and XPM dominates. Perfect phase conjugation is obtained at $L=L_{\mathrm{op}}$ and $L=0.95 L_{\mathrm{op}}$ with and without attenuation and SPM and XPM effects respectively. In all figures, solid lines shows theoretical results with $\alpha=0$ whereas the circles $(\circ)$, squares $(\square)$, diamonds $(\diamond)$ and cross $(\times)$ shows the simulation results with $\alpha=0$ and $\alpha=0.9 \mathrm{~dB} \mathrm{~km}^{-1}$ without and with SPM and XPM effect respectively.

signal (and, idler) wavelength is taken at ZDW of HNLF and dispersion length is calculated to be $1240 \mathrm{~km}$ for dispersion coefficient, $D=0.4 \mathrm{ps} \mathrm{nm}^{-1} \mathrm{~km} \mathrm{[15].}$

We now consider the effects of HNLF length and pump power on phase conjugation.

\subsection{Influence of fiber length}

We studied the effect of length of HNLF on idler evolution for one period length of efficiency, i.e. for $L=2 L_{\mathrm{op}}$ at pumps power of $9.2 \mathrm{dBm}$. Figure 3(a) shows the dependence of idler efficiency as a function of HNLF length for $\alpha=0$ and $\alpha=0.9 \mathrm{~dB} \mathrm{~km}^{-1}$ with and without SPM and XPM effect. As HNLF length $L$ increases, efficiency also increases and a peak efficiency is obtained at optimum fiber length $L_{\mathrm{op}}$ for $\alpha=0$; however, beyond $L_{\text {op }}$ efficiency decreases. At $L=L_{\text {op }}$, a peak 
efficiency of 0.097 was obtained. Simulated values matches closely to the theoretical values for $\alpha=0$. The efficiency decreases significantly, when $\alpha=0.9 \mathrm{~dB} \mathrm{~km}^{-1}$ is taken into the simulation and efficiency-peak shifted to $0.8 L_{\mathrm{op}}$. This shift in efficiency-peak is due to attenuation in pumps and dependence of efficiency on pumps power.

When SPM and XPM effects with $\alpha=0$ is considered in the simulation, efficiency decreases as compare to $\alpha=0$, this is due to increased residual phase mismatch $(\Delta k)$ by the virtue of SPM and XPM. Also, as the interaction length of fiber increases $\left(L>L_{\mathrm{op}}\right)$ the SPM and XPM effect dominates over FWM and so the efficiency decreases. The period of efficiency is decreased due to increased residual phase mismatch by SPM and XPM effect, which is clearly visible in figure 3(a). Finally, we combine the effects of SPM and XPM and attenuation $\left(\alpha=0.9 \mathrm{~dB} \mathrm{~km}^{-1}\right)$ and an efficiency of 0.018 was obtained at $L=L_{\text {op }}$.

Figure 3(b) shows the effect of length of HNLF on the phase-offset. At $L=L_{\mathrm{op}}, \Delta \Phi_{\text {offset }}=0$ and idler phase $\phi_{\mathrm{i}}$ is an exact conjugate of signal phase $\phi_{\mathrm{s}}$ as given in (4) for $\alpha=0$. A non-zero phase-offset is present at all lengths other than $L=L_{\mathrm{op}}$. A small deviation in phase-offset is present at $L<L_{\mathrm{op}}$ when attenuation $\left(\alpha=0.9 \mathrm{~dB} \mathrm{~km}^{-1}\right)$ is considered in simulation. As length of HNLF increased, attenuation is increased and so the phase-offset. This is due to dependence of phase-offset on pumps power. A deviation in phase-offset is present when the SPM and XPM effects are taken into the simulation without attenuation due to phase change in signal, idler and pump waves. Effect of SPM and XPM increases with the fiber length, which results in the increment of phaseoffset. Finally, when effects of SPM and XPM and $\alpha=0.9 \mathrm{~dB} \mathrm{~km}^{-1}$ is considered, the two effects nearly compensate for each other till $L=1.3 L_{\mathrm{op}}$ and a zero phase-offset obtained at $L=0.95 L_{\mathrm{op}}$, which resulted in phase conjugation at $2.85 \mathrm{~km}$ of HNLF length at $9.2 \mathrm{dBm}$ pumps power with efficiency $-17 \mathrm{~dB}$.

\subsection{Influence of pump power}

We studied the effect of pump 1 power on the efficiency and phase-offset by keeping the pump $_{2}$ power constant at $9.22 \mathrm{dBm}$ at HNLF length of $0.95 L_{\mathrm{op}}$. Figure 4(a) shows that efficiency increases with increasing pump $p_{1}$ power for $\alpha=0$. This efficiency decreases considerably when attenuation $\alpha=0.9 \mathrm{~dB} \mathrm{~km}^{-1}$ is taken into the simulation. This is due to attenuation of pumps and signal powers. When we considered SPM and XPM effects, efficiency decreases as compare to efficiency for $\alpha=0$, because of increment in residual phase mismatch $\Delta k$ due to nonlinear self and cross-phase changes introduced in pump $_{1}$ and pump $_{2}$ by SPM and XPM. This increment in residual phase mismatch further increases with increased pump $_{1}$ power causes reduction in efficiency at higher pump ${ }_{1}$ powers. The efficiency further decreases, when the $\alpha=0.9 \mathrm{~dB} \mathrm{~km}^{-1}$ and SPM and XPM effects are taken into the simulation, resulting in 0.02 of efficiency at $9.22 \mathrm{dBm}$

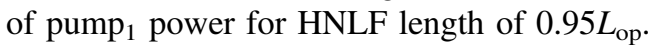

Figure 4(b) shows the effect of pump $_{1}$ power on the phase-offset. At $9.22 \mathrm{dBm}$ of pump p $_{1}$ power the phase-offset is

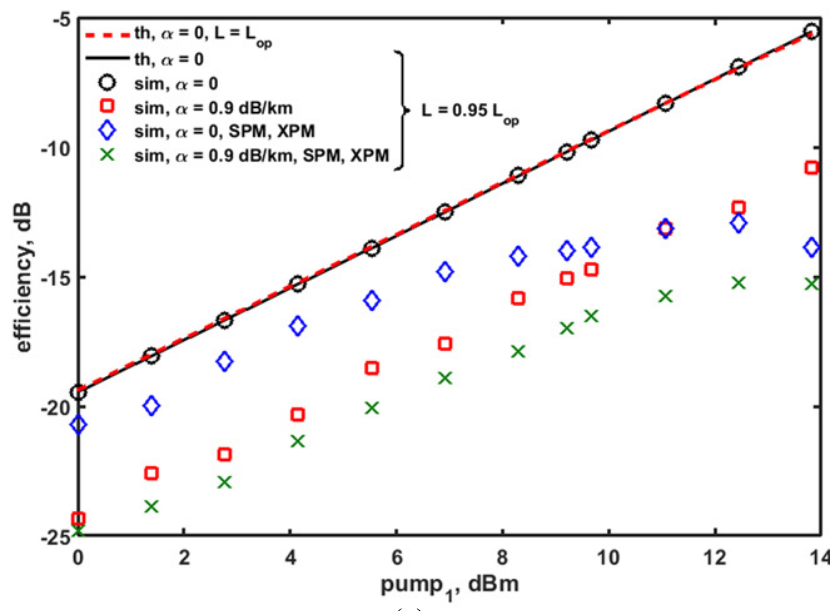

(a)

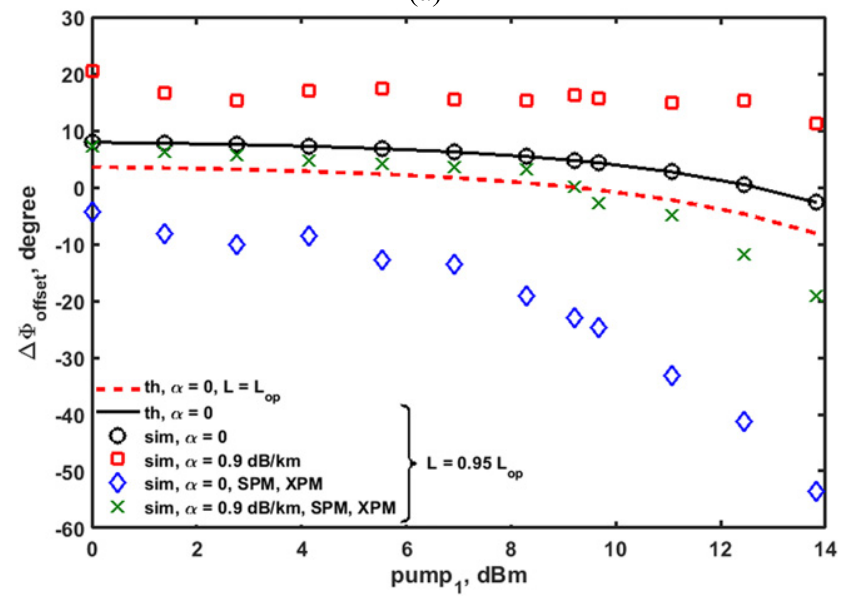

(b)

Figure 4. Effect of pump ${ }_{1}$ power on idler. In figure 4(a), efficiency increase as the pump $p_{1}$ power is increased for $\alpha=0$ and for $\alpha=0.9 \mathrm{~dB} \mathrm{~km}^{-1}$ with SPM and XPM effects, the efficiency is smaller due to attenuation and increase in residual phase mismatch by SPM and XPM effects in pumps. In figure 4(b), variation of phase-offset with pump 1 power is shown. Signal power is kept constant at $-7 \mathrm{dBm}$ and $\mathrm{HNLF}$ length is kept at $0.95 L_{\mathrm{op}}$. The increase in phase-offset due to attnuation is cancelled by decrease in phase-offset by SPM and XPM effects results in perfect phase conjugation at $9.22 \mathrm{dBm}$ of pump $_{1}$ power. In all figures, dash (-) line shows theoretical results with $\alpha=0$ for $L=L_{\mathrm{op}}$ and solid lines shows theoretical results with $\alpha=0$ whereas the circles (o), squares $(\square)$, diamonds $(\diamond)$ and cross $(\times)$ shows the simulation results with $\alpha=0$ and $\alpha=0.9 \mathrm{~dB} \mathrm{~km}^{-1}$ without and with SPM and XPM effect respectively for $L=0.95 L_{\mathrm{op}}$.

almost zero for both theory and simulations at $\alpha=0$ for $L=L_{\mathrm{op}}$ and at $\alpha=0.9 \mathrm{~dB} \mathrm{~km}^{-1}$ with SPM and XPM for $L=0.95 L_{\mathrm{op}}$, confirming the phase conjugation. A non-zero phase-offset is present at all other powers for $\alpha=0$ as expected from (5). With $\alpha=0.9 \mathrm{~dB} \mathrm{~km}^{-1}$, pumps power decreases which results in increase in phase-offset, whereas, when SPM and XPM effects are considered with $\alpha=0$, phase-offset decreases due to increase in residual phase mismatch $\Delta k$. When the effects of attenuation and SPM and XPM are combined, they nearly compensate each other till $10 \mathrm{dBm}$ of pump $_{1}$ power, resulting in perfect phase conjugation at pump 1 power of $9.22 \mathrm{dBm}$. 
Finally, we observed that perfect phase conjugation with efficiency 0.097 is possible at $L=L_{\mathrm{op}}$ with pumps power of $9.22 \mathrm{dBm}$ in theory for $\alpha=0$, whereas, with attenuation $\left(\alpha=0.9 \mathrm{~dB} \mathrm{~km}^{-1}\right)$ and SPM and XPM effects, phase conjugation with efficiency $-17 \mathrm{~dB}$ is predicted at $L=0.95 L_{\mathrm{op}}$ $\sim 2.85 \mathrm{~km}$ with pumps power of $9.22 \mathrm{dBm}$ by simulation.

\section{Conclusion}

In this paper, we have proposed a scheme of frequency-shift free OPC. OPC is realized using counter-propagating dual pump FWM in HNLF, with pump frequencies placed symmetrically about signal frequency. Symmetrical placement of pump frequencies results in generation of idler in the signal band with high efficiency. The idler and signal appears at opposite ends of the HNLF, which then spatially filtered to eliminates the needs of polarization-shift in idler. Further, we assume both pumps to be co-polarized for polarization insensitive FWM. By keeping the pump powers equal, we avoid instability of FWM process in our scheme.

Using NLSE, we derived expressions for signal and idler evolution inside OPC. We showed that for optimum length $L_{\mathrm{op}}$ of HNLF at a given pump power, idler phase is equal to conjugate of signal phase. Numerical solution of OPC equation (2) confirms that idler is phase conjugate of signal.

We further verify the perfect phase conjugation by proposed OPC under ideal lossless condition, by resolving the temporal profile of electric field envelope of signal and idler modulated with repetitive pattern of ' 1101 ' at $40 \mathrm{Gbps}$ with non-zero chirp MZM.

We have identified the critical design parameters: length of HNLF and pump power and analyses their effects on the performance on OPC. In our simulation we considered the effect of SPM and XPM and neglected the stimulated Brillouin and Raman scattering. We have shown that for HNLF length $L<L_{\mathrm{op}}$, FWM prevails over SPM and XPM and SPM and XPM dominates for $L>L_{\mathrm{op}}$. At $L<L_{\mathrm{op}}$, a small phaseoffset is present, which can be avoided by choosing HNLF length $L=0.95 L_{\mathrm{op}}$. We have also shown that effect of SPM and XPM is higher at higher pump powers. This effect of SPM and XPM is nullified by attenuation upto $10 \mathrm{dBm}$ of pump $_{1}$ power. For HNLF length of $2.85 \mathrm{~km}$ and pumps power at $9.22 \mathrm{dBm}$, we observe perfect phase conjugation with efficiency $-17 \mathrm{~dB}$. In practice, efficiency is limited by stimulated Brillouin and Raman scattering process, which was avoided by keeping detuning between pumps and signal away from Brillouin and Raman gain spectrum.

In conclusion, we have proposed a novel scheme for generating frequency-shift free OPC using counter-propagating dual pump FWM in nonlinear fiber. We have shown that under right conditions, idler phase is conjugate of signal phase. Simulations carried out show good agreement with theoretical results.

\section{References}

[1] He Guang S 2002 Optical phase conjugation: principles, techniques, and applications Prog. Quantum Electron. 26 131-91

[2] Vasilyev M and Stelmakh N 2006 Squeezing and fiber-optic communications Int. J. Mod. Phys B 201536

[3] Yariv A, Fekete D and Pepper David M 1979 Compensation for channel dispersion by nonlinear optical phase conjugation Opt. Lett. 4 52-4

[4] Du Liang B, Morshed M M and Lowery Arthur J 2012 Fiber nonlinearity compensation for OFDM super-channels using optical phase conjugation Opt. Express 20 19921-7

[5] Jansen S L, van den Borne, Krummrich P M, Spälter S G, Khoe D and de Waardt H 2006 Long-haul DWDM transmission systems employing optical phase conjugation J. Lightwave Tech. 12 505-20

[6] Janer C L and Connelly M J 2011 Optical phase conjugation technique using four-wave mixing in semiconductor optical amplifier Electron. Lett. 47 716-17

[7] Stephens M F C, Tan M, Phillips I D, Sygletos S, Harper P and Doran N J 2014 1.14 Tb/s DP-QPSK WDM polarization-diverse optical phase conjugation Opt. Express 22 11840-8

[8] Morshed M, Du L B, Foo B, Pelusi M D, Corcoran B and Lowery A J 2014 Experimental demonstrations of dual polarization CO-OFDM using mid-span spectral inversion for nonlinearity compensation Opt. Express 22 10455-66

[9] Xiang-Jun X, Jian-Xin M, Qi Z, Chao-Gong D, Kui-Ru W, Chong-Xiu Y and Bo L $2009155 \mathrm{Mb} / \mathrm{s}$ and $10 \mathrm{~Gb} / \mathrm{s}$ combined FSK-IM/optical label-packet modulation signals $100 \mathrm{~km}$ transmission over standard single mode fiber using mid-span spectral inversion by four-wave mixing in an SOA Chin. Phys. B 18 3449-52

[10] Rong H, Ayotte S, Mathlouthi W and Paniccia M 2008 Midspan dispersion compensation via optical phase conjugation in silicon waveguide $O F C / N F O E C$

[11] Inoue K 1997 Spectral inversion with no wavelength shift based on four-wave mixing with orthogonal pump beams Opt. Lett. 22 1772-4

[12] Corchia A, Antonini C, DOttavi A, Mecozzi A, Martelli F, Spano P, Guekos G and DallAra R 1999 Mid-span spectral inversion without frequency shift for fiber dispersion compensation : a system demonstration IEEE Photonics Technol. Lett. 11 275-8

[13] McKinstrie C J and Radic S 2004 Phase-sensitive amplification in fiber Opt. Express 12 4973-9

[14] Agrawal G P 2013 Nonlinear Fiber Optics 4th edn (New York: Academic)

[15] HNLF-SPINE ZDW 1540 HNLF Non-Linear Fiber Modules (http://fiber-optic-catalog.ofsoptics.com/item/opticalfibers/highly-nonlinear-fiber-optical-fibers 1/hnlf-spinezdw-1540)

[16] Liu X, Zhou X and Lu C 2005 Multiple four-wave mixing selfstability in optical fibers Phys. Rev. A 72013811

[17] Liu X, Yang X, Lu F, Ng J, Zhou X and Lu C 2004 Stable and uniform dual-wavelength erbium-doped fiber laser based on fiber Bragg gratings and photonic crystal fiber Opt. Express 13 142-7

[18] Bravetti P, Ghislotti G and Balsamo S 2004 Chirp-inducing mechanisms in Mach-Zehnder modulators and their effect on $10 \mathrm{~Gb} / \mathrm{s}$ NRZ transmission studied using tunable-chirp single drive devices J. Lightwave Tech. 22 605-11 